bedside or occasionally with the patient in a separate room out of earshot of other patients. - I am, etc., Queen Elizabeth Hospital,
Birmingham 15 .

Clifford Hawkins.

\section{Group A Streptococci Resistant to Lincomycin}

SIR,-We have isolated group A streptococci resistant to lincomycin, erythromycin, and oleandomycin from patients under treatment with lincomycin.

Early in August 1967 group A streptococci sensitive to penicillin, tetracycline, erythromycin, and lincomycin were isolated from patients in the burns unit at Queen Mary's Hospital, Roehampton. Pencillinaseforming staphylococci were widespread in the burns, and penicillin proved ineffective in eliminating the streptococci, so lincomycin was given to those still infected. Four weeks later, after it had been given to 12 patients, group A streptococci resistant to lincomycin, erythromycin, and oleandomycin were isolated from five patients, all of whom had received lincomycin, and from one nurse. All patients in the ward were then given a full course of treatment with cloxacillin, and the streptococcal infection was eliminated within 10 days.

In all, group A streptococci were isolated from 21 patients and four nurses. One patient produced a rash suggestive of scarlet fever, but all the others were symptomlessly infected.

The resistant streptococci were first recognized by disc diffusion tests. In tube-sensitivity tests with heavy inocula they grew in the presence of $100 \mu \mathrm{g} . / \mathrm{ml}$. of each of the three antibiotics; sensitive organisms were inhibited by $0.4 \mu \mathrm{g} . / \mathrm{ml}$. or less. Six cultures were typedfour resistant and two sensitive-all belonged to type 4

We are not aware of previous reports of lincomycin-resistant group A streptococci. In 1959 Lowbury and Hurst ${ }^{2}$ isolated cultures resistant to erythromycin and oleandomycin from the burns of patients treated with erythromycin, but there is not yet any evidence from surveys carried out at Colindale that erythromycin resistance is widespread outside burns units.

In the present outbreak intensive use of lincomycin quickly resulted in the emergence of lincomycin-resistant streptococci which showed cross-resistance to the chemically unrelated macrolide antibiotics erythromycin and oleandomycin, though erythromycin had not been used in the unit for some months. A similar antibiotic cross-resistance has been seen in pneumococci ${ }^{23}$ and in one type of macrolide resistance in Staphlyococcus

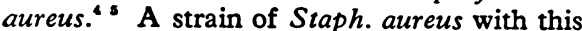
resistance was prevalent in our unit, and several patients were concurrently infected with erythromycin/lincomycin-resistant staphylococci and streptococci.

Tetracycline resistance among group A streptococci is now common, and this antibiotic has no place in the treatment of streptococcal infections. Should linked resistance to erythromycin and lincomycin become more common the consequences might be serious, because these antibiotics are regarded as the drugs of choice for the treatment of streptococcal infection in patients with a

We wish to thank Mr. A. J. Evans for permission to publish information about patients under his care.

Department of Pathology,

Queen Mary's Hospital

Roehampton, London S.W.15.

J. H. HewitT

Cross-infection Reference

Colindale, London N.W.9.

\section{REPERENCES} ${ }^{1}$ Lowbury, E. J. L., and Hurst, L., f. clin. Path., 852 .

- Dixon, J. M. S., Lancet, 1967, 1, 573 . F., 1964, 2,603 .

, and Mitsuhashi, S. fap. F. Microbiol., 1966, 10, 59.

\section{Transplant Lung}

SIR,-Dr. J. M. B. Hughes and Mr. W. J. Dempster (27 January, p. 250) are understandably disappointed with the incompleteness of the evidence presented by Mr. M. Slapak and others (13 January, p. 80) for the existence of the condition of transplant lung. However, that it should not be dismissed on this account is suggested by our experience with a similar case.

A 21-year-old man with chronic renal failure due to membranous glomerulonephritis underwent cadaveric kidney transplantation on 11 October 1967. The postoperative course is shown in the Figure. Early progress was satisfactory and on the 37 th day creatinine clearance was $46 \mathrm{ml}$./min. Pyrexia was noted during the next 15 days and the creatinine clearance gradually fell to $27 \mathrm{ml} . / \mathrm{min}$. 60 days after operation. Kidney function improved following an increased dose of prednisone.

On the 80 th postoperative day pyrexia recurred and was accompanied on this occasion by gradually increasing breathlessness, for which no infective cause was found. Clinical examination revealed a few crepitations at the lung bases together with evidence of pulmonary hypertension. Chest $x$-rays, which had previously been normal, now showed minimal bilateral pulmonary infiltrates which increased during the next six history of penicillin allergy.

-We are, etc., C. A. M. FRASER.

Kislak, J. W., New Engl. f. Med., 1967, 276

days. Arterial $\mathrm{Po}_{2}$ levels were reduced (50-60 $\mathrm{mm}$. $\mathrm{Hg}$ ), and $\mathrm{PCO}_{3}$ was normal. The fever responded within 12 hours to an increase in prednisone dosage given on the 86th day, and the breathlessness improved; a further chest $x$ ray on the 88th postoperative day showed considerable clearing of the lung fields.

Formal tests of lung function were performed 91 days after operation (see Table). These

Tests of Lung Function on 91 st Postoperative Day

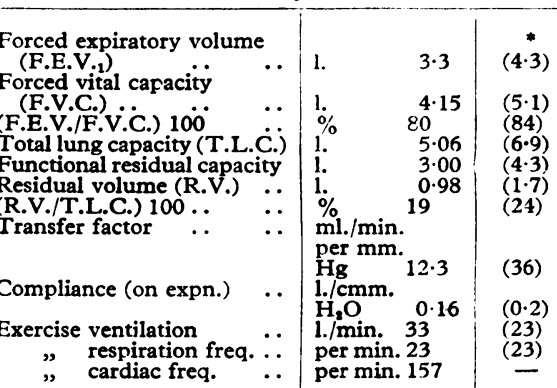

* Average values for a man the age and size of the patient.

showed reductions in the vital capacity and the compliance to about three-quarters of the expected values, with proportional changes in the forced expiratory volume and the residual volume. The transfer factor was reduced to about one-third of expected and the ventilation on exercise was increased. These results and the associated hypoxaemia are evidence for a defect of gas transfer. They were obtained at a time when the kidney function was relatively good. Subsequently, after further episodes of pyrexia the creatinine clearance fell to $10 \mathrm{ml}$ / min., but without any recurrence of symptoms referable to the chest. Renal biopsy on the 112 th day showed histological evidence of rejection. The dose of prednisone has again been increased and renal function is now improving.

In summary, this patient has had two episodes of graft rejection, and the second of these followed an episode of respiratory embarrassment which bore a striking resemblance to the condition of "transplant lung" described by Mr. Slapak and his colleagues. The syndrome coincided with a reduction of prednisone dosage and improved rapidly when this was increased. This response would seem to exclude cryptogenic pulmonary infection as the cause of the pulmonary lesion, but its

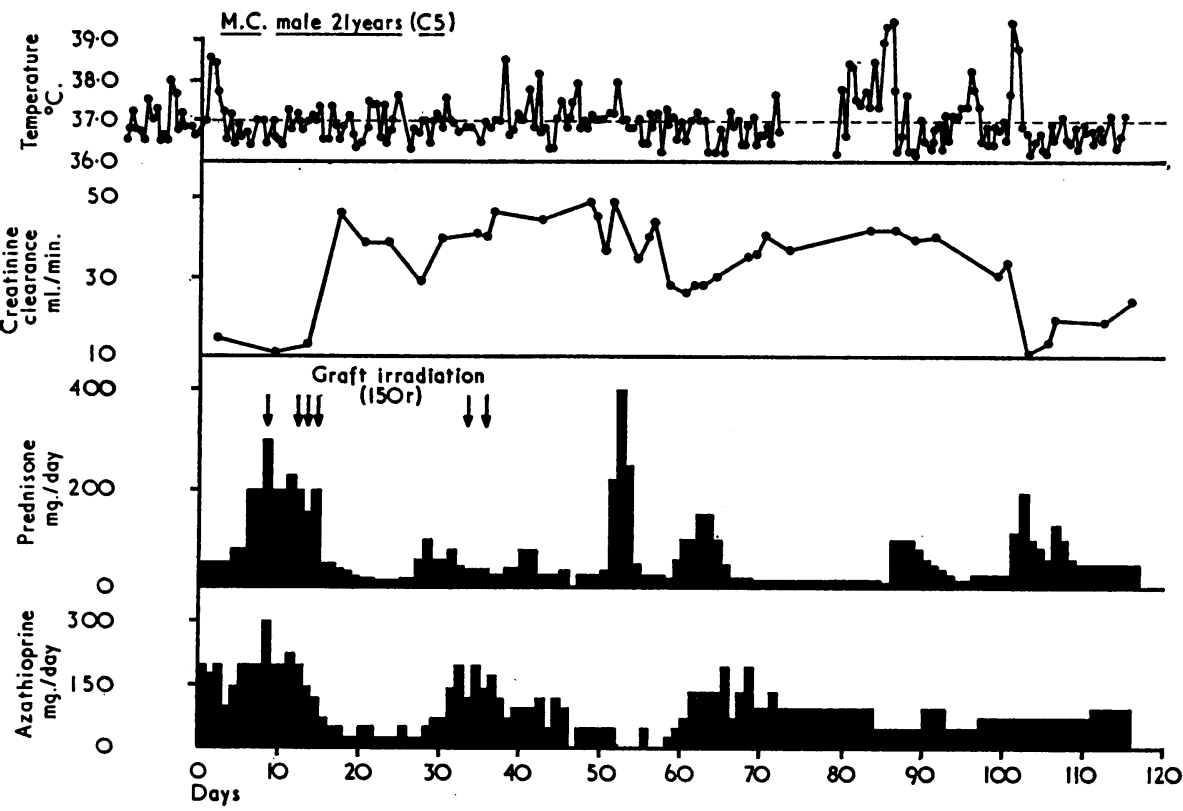

